\title{
easyAmber: a step away from inefficient "static" approaches towards a deeper understanding of protein dynamics
}

\author{
Dmitry Suplatov \\ Belozersky Institute of \\ Physicochemical Biology, \\ Lomonosov Moscow State University, \\ Moscow, Russia \\ d.a.suplatov@belozersky.msu.ru
}

\author{
Yana Sharapova \\ Belozersky Institute of \\ Physicochemical Biology, \\ Lomonosov Moscow State University, \\ Moscow, Russia \\ sharapova@belozersky.msu.ru
}

\author{
Vytas Švedas \\ Belozersky Institute of \\ Physicochemical Biology and Faculty \\ of Bioengineering and Bioinformatics, \\ Lomonosov Moscow State University, \\ Moscow, Russia \\ vytas@belozersky.msu.ru
}

\begin{abstract}
Molecular dynamics (MD) can improve the success rate of in silico tools for drug discovery and protein engineering by accounting for protein structure flexibility/plasticity and predicting the time-dependent behavior of a molecular system, but requires specialized training and skills, what impedes practical use by many investigators. We have developed easyAmber - a comprehensive toolbox to automate the MD routines implemented in the Amber package, freely available
\end{abstract}

https://biokinet.belozersky.msu.ru/easyAmber.

Keywords - structural biology, molecular dynamics, protein structure flexibility, time-dependent behavior, protein-ligand interaction, computational drug discovery

\section{Motivation and aim}

\section{Motivation}

Computational biology is overwhelmed by "static" approaches that take into account only a single PDB entry of the protein of interest, e.g., to select hot-spot positions to improve its biocatalytic function or design selective inhibitors as prototypes of novel drugs. Despite some positive progress, the overall success rates of these approaches remain disappointingly low, probably because protein structures are flexible and the corresponding ensemble of conformations has to be explicitly taken into account to understand the structurefunction relationship. Molecular dynamics (MD) can address the challenge of accounting for protein flexibility, but presents a very complex method that requires specialized training and advanced computer experience, thus significantly limiting its everyday use.

Aim

The aim was to develop comprehensive and easy-to-use assist software to automate the simulation workflow and thus improve the user's experience with MD.

\section{Results}

We have developed the easyAmber - a set of wrapper scripts as a supplement to the Amber suite to assist the MD simulation starting from the 3D-model of a protein/proteinligand complex. The autoligand.pl master-script assists the preparation of the Amber parameter files for a custom lowmolecular-weight ligand. The automodel.pl master-script assists the preparation of a full-atom molecular system in water. The autorun.pl master-script accommodates the seven-step MD simulation pipeline: the initial optimization of the molecular system (i.e., three-step energy minimization with water relaxation), heating (in the NVT ensemble), equilibration (in the NPT ensemble), followed by the classical/conventional MD (in the NVT ensemble), and optionally concluded by the accelerated MD simulation (in the NVT ensemble). Thus, two major MD methods are supported - the classical and accelerated MD - that together can help to assess protein structure flexibility on a wide range of timescales.

The easyAmber implements advanced MD protocols, but is easy-to-operate on a personal desktop station equipped with a compatible gaming GPU-accelerator, as well as a powerful supercomputer, thus significantly increasing productivity. The new toolkit was used in recent studies of conformational plasticity in NanA from S.pneumoniae and human p38 $\alpha$ MAPK, both featuring well-known targets for drug design, to operate hundreds of independent trajectories at various settings worth tens of microseconds, using various HPC resources [1-3]. A step-by-step practical tutorial to the easyAmber was published in the corresponding paper [4], with supplementary technical documentation available on-line at https://biokinet.belozersky.msu.ru/easyAmber\#techdoc. We hope that easyAmber will contribute to a more widespread use of MD in a daily laboratory routine, supporting a recent trend away from inefficient "static" approaches in protein sciences towards a deeper understanding of structural dynamics, leading to a more efficient selection of hot-spots for protein engineering and complementary ligands/inhibitors by companion in silico tools.

\section{ACKNOWLEDGMENT}

The study was funded by Russian Foundation for Basic Research according to the research project (19-04-01297). The use of HPC resources at the Lomonosov Moscow State University supported by the project RFMEFI62117X0011 is acknowledged [5].

\section{REFERENCES}

[1] Sharapova Y., Suplatov D., Švedas V. (2018) Neuraminidase A from Streptococcus pneumoniae has a modular organization of catalytic and lectin domains separated by a flexible linker. FEBS J. 285(13):24282445.

[2] Suplatov D., Kopylov K., Sharapova Y., Švedas V. (2019) Human p38 $\alpha$ Mitogen-Activated Protein Kinase in the Asp168-Phe169Gly170-in (DFG-in) state can bind allosteric inhibitor Doramapimod. J. Biomol Struct. Dyn. 37(8): 2049-2060.

[3] Sharapova Y., Suplatov D., Švedas V. (2018) Simulating the Longtimescale Structural Behavior of Bacterial and Influenza Neuraminidases with Different HPC Resources. Supercomput. Front. Innov. 5(3): 30-33.

[4] Suplatov D., Sharapova Y., Švedas V. (2020) easyAmber: a comprehensive toolbox to automate the molecular dynamics simulation of proteins. J. Bioinf. Comput. Biol. in press

[5] Sadovnichy V., Tikhonravov A., Voevodin V., Opanasenko V. (2013) Lomonosov: supercomputing at moscow state university. In Vetter J. (ed.) Contemporary High Performance Computing: From Petascale Toward Exascale (Chapman \& Hall/CRC Computational Science). Boca Raton, CRC Press, 283-307. 